\title{
Time-Skew Hebb Rule in a Nonisopotential Neuron
}

\author{
Barak A. Pearlmutter* \\ To appear (1995) in Neural Computation, 7(4) 706-712
}

\begin{abstract}
In an isopotential neuron with rapid response, it has been shown that the receptive fields formed by Hebbian synaptic modulation depend on the principal eigenspace of $\mathbf{Q}(0)$, the input autocorrelation matrix, where $Q_{i j}(\tau)=\left\langle\xi_{i}(t) \xi_{j}(t-\tau)\right\rangle$ and $\xi_{i}(t)$ is the input to synapse $i$ at time $t$ (Oja 1982). We relax the assumption of isopotentiality, introduce a time-skewed Hebb rule, and find that the dynamics of synaptic evolution are determined by the principal eigenspace of $\widehat{\mathbf{Q}}$. This matrix is defined by $\widehat{Q}_{i j}=\int_{0}^{\infty}\left(Q_{i j} * \psi_{i}\right)(\tau) K_{i j}(\tau) d \tau$, where $K_{i j}(\tau)$ is the neuron's voltage response to a unit current injection at synapse $j$ as measured $\tau$ seconds later at synapse $i$, and $\psi_{i}(\tau)$ is the time course of the opportunity for modulation of synapse $i$ following the arrival of a pre-synaptic action potential.
\end{abstract}

\section{Introduction}

Hebbian synaptic modification involves the enhancement of synaptic efficacy in response to simultaneous pre- and post-synaptic activity. This form of learning has taken on particular importance with its discovery in various parts of the brain, a prominent example being long-term potentiation (LTP) in the hippocampus (Bliss and Collingridge 1993).

The classic Oja (1982) analysis of the temporal evolution of Hebbian synapses assumes dependence on instantaneous conjunction of pre- and postsynaptic activity and a linear neuron with an isopotential membrane which responds quickly compared to the time course of its inputs. However, it is believed that the trans-membrane potential in cortical neurons can vary widely across the dendritic tree on a time scale important to LTP (Koch et al. 1982; Rall 1977; Zador et al. 1995). It also appears that LTP occurs when postsynaptic voltage is elevated during a finite time window following presynaptic activation (Bliss and Collingridge 1993). This paper analyzes the effects of Hebbian learning under these conditions, and grew out of an attempt to understand the clustered spatial structure of facilitated Hebbian synapses seen in the model nonisopotential neurons of Brown et al. (1991) and Mel (1992). Even though linearity in Hebbian systems can be less restrictive than one might suppose (Miller 1990), it is important to emphasize that the analysis here concerns a neuron that is nonisopotential but nonetheless linear. Nonlinear channels and saturation ${ }^{1}$ are not considered, although they likely play an important computational role (Mel 1993).

\footnotetext{
${ }^{*}$ Siemens Corporate Research, 755 College Road East, Princeton, NJ 08540, bap@ learning.scr.siemens.com

${ }^{1}$ We model synaptic events as current injections, even though it would be more faithful to the biology to use conductance changes. Unfortunately, using conductance chances instead of current injection introduces the solution of a general causal linear integral equation into the calculation of $\widehat{\mathbf{Q}}$.
} 


\section{Results}

In Oja (1982) and its extensions, the post-synaptic potential $V(t)$ is shared across all synapses, and the input signal $\xi(t)$ plays two roles: that of presynaptic activity in $V(t)=\sum_{j} w_{j} \xi_{j}(t)$, where $w_{j}$ is the efficacy of synapse $j$, and that of the window of opportunity for synaptic facilitation in $d w_{i} / d t=\eta \xi_{i}(t) V(t)$ - decay, where the learning rate $\eta>0$ is a constant of proportionality. Taking the expected value, this leads to an expected dynamics of $\langle d \mathbf{w} / d t\rangle=\eta \mathbf{Q}(0) \mathbf{w}-\langle\mathbf{d e c a y}\rangle$, where $Q_{i j}(\tau)=\left\langle\xi_{i}(t) \xi_{j}(t-\tau)\right\rangle$. These expected dynamics approximate the true dynamics well when $\eta$ is small. The synaptic evolution is therefore governed ${ }^{2}$ by the principal eigenvectors of $\mathbf{Q}(0)$.

Here, we allow the neuron to be nonisopotential with

$$
V_{i}(t)=\sum_{j} w_{j} \int_{0}^{\infty} \xi_{j}(t-\tau) K_{i j}(\tau) d \tau=\sum_{j} w_{j}\left(\xi_{j} * K_{i j}\right)(t)
$$

where $V_{i}(t)$ is the post-synaptic potential at synapse $i, K_{i j}(\tau)$ gives the voltage response of the neuron to a unit current injection at synapse $j$ as measured $\tau$ seconds later at synapse $i$, and functional convolution is defined by $(f * g)(t)=\int_{0}^{\infty} f(t-\tau) g(\tau) d \tau$. The transfer impedence matrix $\mathbf{K}$ (Koch et al. 1982) is easy to calculate and reason about in the temporal frequency domain (Zador et al. 1991, 1993, 1995).

We also generalize the Hebb rule by distinguishing between $\xi_{i}(t)$, the presynapic input ${ }^{3}$ to synapse $i$ at time $t$, and $\psi_{i}(\tau)$, the window of opportunity for synaptic modulation following presynaptic activity at synapse $i$. The synaptic modulation equation thus becomes

$$
\begin{aligned}
\frac{d w_{i}}{d t}(t) & =\eta \int_{0}^{\infty} \xi_{i}(t-\tau) \psi_{i}(\tau) d \tau V_{i}(t)-\text { decay } \\
& =\eta\left(\xi_{i} * \psi_{i}\right)(t) V_{i}(t)-\text { decay } \\
\left\langle\frac{d w_{i}}{d t}\right\rangle & =\eta \sum_{j} w_{j}\left\langle\left(\xi_{i} * \psi_{i}\right)\left(\xi_{j} * K_{i j}\right)\right\rangle-\langle\text { decay }\rangle \\
& =\eta \sum_{j} w_{j} \int_{0}^{\infty} K_{i j}(\tau)\left(Q_{i j} * \psi_{i}\right)(\tau) d \tau-\langle\text { decay }\rangle
\end{aligned}
$$

If we define the matrix $\widehat{\mathbf{Q}}$ by

$$
\widehat{Q}_{i j}=\int_{0}^{\infty} K_{i j}(\tau)\left(Q_{i j} * \psi_{i}\right)(\tau) d \tau
$$

then, in matrix notation, we obtain the familiar

$$
\left\langle\frac{d \mathbf{w}}{d t}\right\rangle=\eta \widehat{\mathbf{Q}} \mathbf{w}-\langle\mathbf{d e c a y}\rangle
$$

\footnotetext{
${ }^{2}$ When using a simple Hebb rule in a linear neuron, it is necessary to invoke an additional mechanism to prevent runaway synaptic facilitation. The particular decay term used has a strong effect, an issue explored in Oja (1982), Miller and MacKay (1994), and Goodhill and Barrow (1994). In essence, with a multipicative decay term, w evolves to the principal eigenvector of $\mathbf{Q}(0)$ regardless of its initial value, whereas subtractive decay terms cause $\mathbf{w}$ to evolve towards principal eigenvectors of $\mathbf{Q}(0)$ but also tend towards the corners of the space.

${ }^{3}$ To speak of it concretely, $\xi_{i}(t)$ typically consists of a series of delta pulses of constant magnitude representing the arrival of action potentials at the presynaptic terminal. Presynaptic changes do not affect $\xi_{i}(t)$-rather, $w_{i}$ encapsulates all the synaptic efficacy, regardless of whether changes to that efficacy are implemented by pre- or post-synaptic physical mechanisms.
} 
where $\widehat{\mathbf{Q}}$ has replaced the usual instantaneous autocorrelation matrix $\mathbf{Q}(0)$. Thus, the evolution of $\mathbf{w}$ is determined by the principal eigenspace of $\widehat{\mathbf{Q}}$ in exactly the same way that, in the classic case, its evolution is determined by the the principal eigenspace of $\mathbf{Q}(0)$. Note that unlike $\mathbf{Q}(0)$, in general $\widehat{\mathbf{Q}}$ is not symmetric. However, $\widehat{\mathbf{Q}}$ takes on simplified forms under important special cases, such as when $\psi_{i}$ is a simple delay, when the neuron is isopotential, or when the neuron's response time is fast compared to the time course of its input.

\section{Application}

Given known electrotonic structure, known correlational structure of the input, and known conditions under which synapses are modulated, one can calculate $\widehat{\mathbf{Q}}$, and thus it's principal components, and thereby predict the receptive field patterns that will be stabilized. When the input and electrotonic structure are particularly simple, it becomes possible to calculate the principal components of $\widehat{\mathbf{Q}}$ analytically. (Broad-band synaptic input with $1 / f^{d}$ correlational structure in both time and space impinging on either an infinite cable or an infinite sheet makes $\widehat{\mathbf{Q}}$ 's principal eigenvectors particularly tractable.) Thus one can predict the formation of clusters, and their length scale, in a manner similar that used by Miller, Keller, and Stryker (1989) to account for receptive fields in the visual system, and precisely analogous to that used by Chernjavski and Moody (1990) to predict the length scale of cortical columns. This is similar in spirit to Mainen et al.'s (1991) account of synapse segregation on nonisopotential neurons, but operates analytically, without laborious simulation. Unfortunately, by tuning within the region of physiological parameter space consistent with experimental data, it is possible to obtain almost any length scale.

We will therefore apply the technique to a simpler situation, where it predicts a robust qualitative effect. In figure 1 a simple model neuron is constructed. The two synapses are given uncorrelated spike trains for input. We let $\psi_{1}=\psi_{2}$ be a square wave of duration comparable to the interspike interval. The elements $Q_{i j} * \psi$ are therefore all equal. This means that, up to a constant,

$\widehat{\mathbf{Q}}=\int_{0}^{\infty} \mathbf{K}(\tau) d \tau$. This matrix is trivially computed, since $\widehat{Q}_{i j}$ is simply the steady-state voltage at synapse $i$ in response to a constant unit current injected at synapse $j$. The two elements of the principal eigenvector of this matrix predict the equilibrium values of the two synaptic weights. In plotting these two synaptic weights as a function of the diameter of the soma (figure 2) we notice an interesting effect: when the soma is absent $(D=0)$ the symmetry of the situation is unbroken, so the two synaptic weights converge to the same value. As $D$ is raised, the soma begins to act as a current sink. This sink is more effective at the more proximal synapse, where the Hebb rule becomes less effective due to the lowered postsynaptic potential. This results in a strengthening of the distal synapse at the expense of the proximal one.

The effect levels off, because even were the soma a perfect current sink, the dendritic resistance $R_{23}$ holds potential in the proximal dendritic compartment long enough for some synaptic modulation to take place.

\section{Summary and Conclusion}

We have shown that passive nonisopotential time-skewed Hebbian learning is mathematically analogous to the isopotential instantaneous case, in that both take the form of an iterated linear operator, plus a decay term, $\langle d \mathbf{w} / d t\rangle=\eta \widehat{\mathbf{Q}} \mathbf{w}-\langle\mathbf{d e c a y}\rangle$. The destiny of $\mathbf{w}$ is thus determined by the principal eigenspace of $\widehat{\mathbf{Q}}$ and the nature of the decay term, and techniques that have been applied to the 
classic case can now be applied to the somewhat more realistic neurons and Hebb rule discussed here.

\section{Acknowledgments}

I would like to thank Ted Carnevale, Uzi Levin, Zach Mainen, Bartlett Mel, John Moody, and especially Tony Zador and Ken Miller for helpful comments and suggestions. Subsequent to submission of this paper, I found that results similar to those in Pearlmutter and Brown (1992), a preliminary abstract of this paper, had been independently discovered by John E. Moody and Alex Chernjavski in 1989, but were not published. Portions of this work were supported by grant ONR 067-32123-332 to Thomas H. Brown and by grants NSF ECS-9114333 and ONR N00014-92-J-4062 to John Moody.

\section{References}

Bliss, T. V. and Collingridge, G. L. (1993). A Synaptic Model of Memory: Long-Term Potentiation in the Hippocampus. Nature, 361(6407), 31-39.

Brown, T. H., Mainen, Z. F., Zador, A. M., and Claiborne, B. J. (1991). Self-Organization of Hebbian Synapses in Hippocampal Neurons. In Lippmann, R. P., Moody, J. E., and Touretzky, D. S. (Eds.), Advances in Neural Information Processing Systems 3, pp. 39-45. Morgan Kaufmann.

Chernjavski, A. and Moody, J. E. (1990). Spontaneous Development of Modularity in Simple Cortical Models. Neural Computation, 2(3), 334-354.

Goodhill, G. J. and Barrow, H. G. (1994). The Role of Weight Normalization in Competitive Learning. Neural Computation, 6(2), 255-269.

Koch, C., Poggio, T., and Torre, V. (1982). Retinal Ganglion Cells: a Functional Interpretation of Dendritic Morphology. Proc. of the Royal Soc. of London B, 298, 227-264.

Mainen, Z. F., Claiborne, B. J., and Brown, T. H. (1991). A Novel Role for Synaptic Competition in the Development of Cortical Lamination. Society for Neuroscience Abstracts, 17(303.6), 759.

Mel, B. W. (1993). Synaptic Integration in an Excitable Dendritic Tree. Journal of Neurophysiology, 70(3), 1086-1101.

Mel, B. W. (1992). NMDA-Based Pattern Discrimination in a Modeled Cortical Neuron. Neural Computation, 4(4), 502-516.

Miller, K. D., Keller, J. B., and Stryker, M. P. (1989). Ocular Dominance Column Development: Analysis and Simulation. Science, 245, 605-615.

Miller, K. D. (1990). Derivation of Linear Hebbian Equations from a Nonlinear Hebbian Model of Synaptic Plasticity. Neural Computation, 2(3), 321-333.

Miller, K. D. and MacKay, D. J. C. (1994). The Role of Constraints in Hebbian Learning. Neural Computation, 6(1), 100-126.

Oja, E. (1982). A Simplified Neuron Model as a Principal Component Analyzer. Journal of Mathematical Biology, 15, 267-273. 
Pearlmutter, B. A. and Brown, T. H. (1992). Hebbian Learning is Jointly Controlled by Electrotonic and Input Structure. Society for Neuroscience Abstracts, 18(567.23).

Rall, W. (1977). Core Conductor Theory and Cable Properties of Neurons. In Kandel, E. R. (Ed.), Handbook of Physiology, Vol. 1, pp. 39-98. Amer. Physiol. Soc., Bethesda.

Zador, A. M., Claiborne, B. J., and Brown, T. H. (1991). Attenuation Transforms of Hippocampal Neurons. Society for Neuroscience Abstracts, 17(605.6), 1515.

Zador, A. M., Hagai, A.-S., and Segev, I. (1995). The Morphoelectrotonic Transform: A Graphical Approach to Dendritic Function. Journal of Neuroscience, 15(4), 1669-1682.

Zador, A. M. and Pearlmutter, B. A. (1993). Efficient Computation of Sparse Elements of the Inverse of a Sparse Near-Tridiagonal Matrix with Application to the Nerve Equation. Tech. rep. OGI-CSE-93-003, Oregon Graduate Institute of Science \& Technology, Department of Computer Science and Engineering, Portland, OR. Ftp cse.ogi.edu: /pub/tech-reports/1993/93003.ps.gz. 

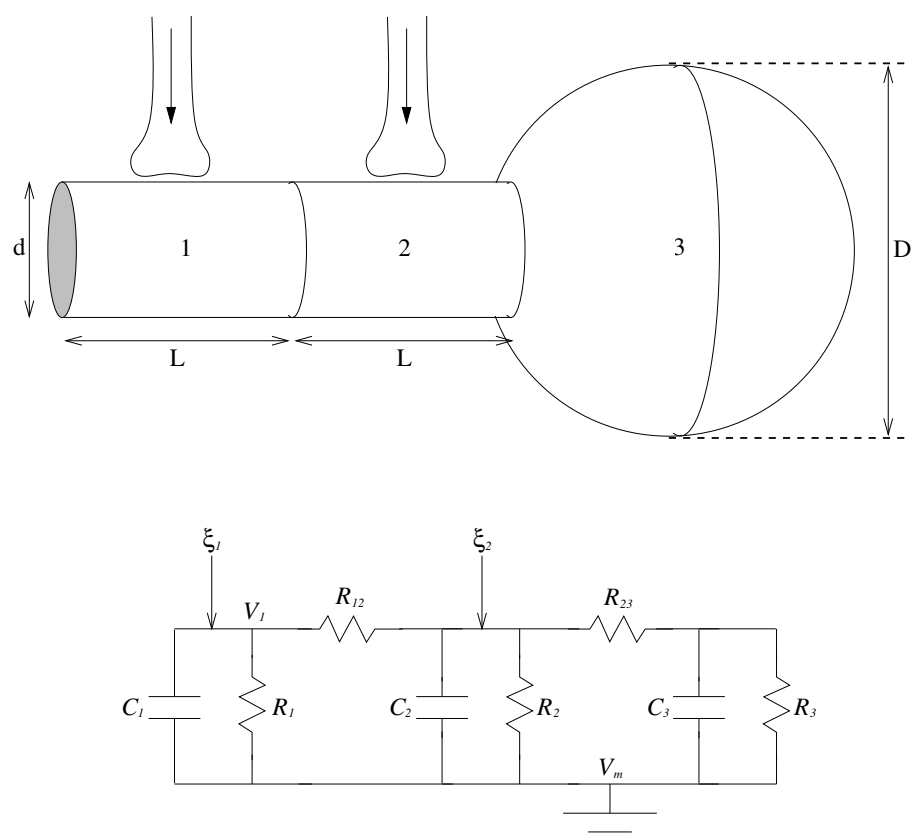

Figure 1: A three-compartment model neuron with two synaptic inputs. The equivalent circuit parameters are therefore $C_{1}=C_{2}=\pi d L C_{m}, C_{3}=\pi D^{2} C_{m}, R_{1}=R_{2}=R_{m} / \pi d L, R_{3}=R_{m} / \pi D^{2}$, $R_{12}=R_{23}=4 R_{a} L / \pi D^{2}$. Membrane parameters used were $C_{m}=1 \mu F / \mathrm{cm}^{2}, R_{m}=50 \mathrm{k} \Omega \mathrm{cm}^{2}$, $R_{a}=200 \Omega \mathrm{cm}, d=2 \mu \mathrm{m}, L=100 \mu \mathrm{m}$. $D$ was allowed to vary.

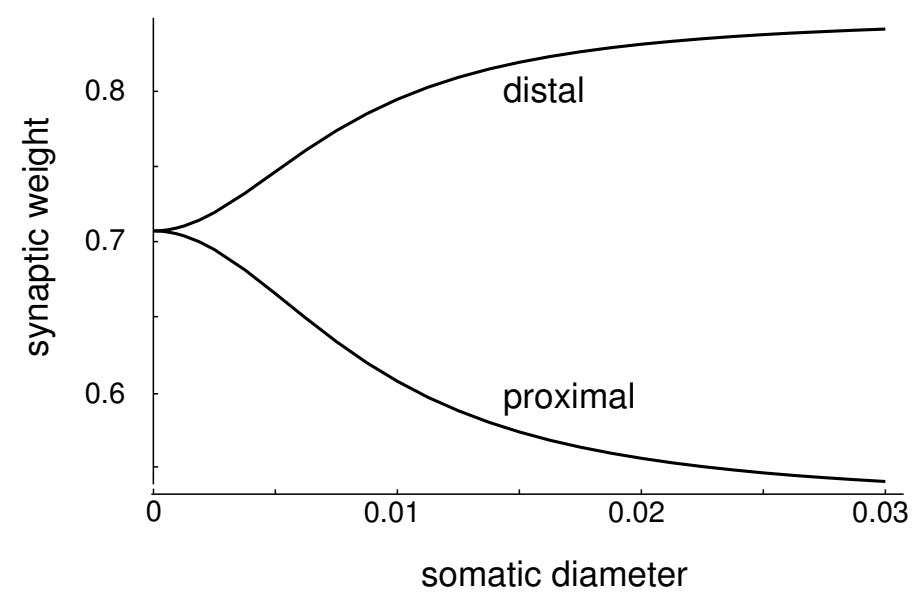

Figure 2: The distal (upper) and proximal (lower) final synaptic weights as a function of $D$, the somatic diameter in $\mathrm{cm}$. These predicted final weights exactly match the final weights evolved in simulations of this process, which were conducted at $D=0,0.002,0.004,0.01 \mathrm{~cm}$. 